\title{
Sécurité alimentaire et durabilité urbaine des modèles alimentaires alternatifs: analyse multicritère basée sur les objectifs de développement durable et l'aménagement urbain durable
}

\author{
Houda Saci ${ }^{*}$ (D) et Ewa Berezowska-Azzag (D) \\ École Polytechnique d'Architecture et d'Urbanisme (EPAU), Alger, Algérie
}

\begin{abstract}
Résumé - Cet article présente une évaluation théorique de la performance urbaine des modèles alimentaires alternatifs dans la prise en charge des objectifs de développement durable (ODD) en vue d'améliorer la sécurité alimentaire urbaine. Elle concerne les modèles des systèmes alimentaires les plus relatés dans la littérature, à savoir le système alimentaire territorialisé (SAT), le City-Region Food System (CRFS) et le système alimentaire urbain intelligent (SAUI) en émergence. Ce travail s'ancre dans le champ du développement urbain durable et pose comme hypothèse l'existence d'une corrélation entre la proximité urbaine des modèles alimentaires alternatifs et leur performance dans l'intégration des objectifs de développement durable. L'évaluation est basée sur une analyse multicritère à scores qui croise deux approches évaluatives déclinées en cibles: une approche normative par les objectifs de développement durable et une autre opérationnelle par l'aménagement urbain durable (AUD). La méthode proposée se veut holistique et systémique contrairement aux méthodes d'évaluation de durabilité agricole existantes. Les scores sont interprétés par des taux d'adéquation des cibles de l'aménagement urbain durable aux objectifs de développement durable et d'appropriation de ces derniers par les domaines de l'écosystème urbain. Les résultats montrent des profils de durabilité des modèles laissant supposer une complémentarité fonctionnelle entre eux. Cependant, c'est le système alimentaire urbain intelligent qui serait le modèle le plus performant avec des taux supérieurs au seuil minimum global de durabilité, suivi par le City-Region Food System, ce qui confirme notre hypothèse de départ. La discussion des résultats pointe les limites de l'évaluation en termes d'objectivité de l'approche, de contextualisation de l'analyse et des contraintes d'applicabilité du système alimentaire urbain intelligent. Enfin, l'étude ouvre une perspective de recherche sur la modélisation d'un système alimentaire urbain intelligent opérationnel, dont ce travail constitue le premier pas.
\end{abstract}

Mots clés : systèmes alimentaires alternatifs / sécurité alimentaire urbaine / objectifs de développement durable / aménagement urbain durable / analyse multicritère

\begin{abstract}
Food security and urban sustainability of alternative food models: Multicriteria analysis based on Sustainable Development Goals and Sustainable Urban Planning. This paper presents a theoretical assessment of alternative food models performance in implementing Sustainable Development Goals (SDG) to improve urban food security. It addresses the three most widely related models in the food systems' literature: The Territorialized Food System, the City-Region Food System (CRFS), and the emerging Smart Urban Food System (SUFS). Nested in the sustainable urban planning field, it assumes that a correlation exists between the urban proximity of the alternative food models and their performance in integrating the Sustainable Development Goals. The valuation is based on a multi-criteria analysis which crosses two approaches: a normative one by Sustainable Development Goals and an operational one by sustainable urban planning. The proposed method is intended to be holistic and systemic unlike existing agricultural sustainability assessment methods. Scores are interpreted by rates of adequacy of sustainable urban planning targets to the Sustainable Development Goals and of appropriation of the latter by the urban ecosystem domains. Results show different profiles of models, suggesting a functional complementarity between them. However, the Smart Urban Food System would be the best performing model with rates
\end{abstract}

*Auteur de correspondance $:$ h.saci@epau-alger.edu.dz 
higher than the global minimum sustainability threshold. It is followed by the City-Region Food System, confirming then our initial hypothesis. Results discussion points out the limits of the evaluation in terms of the approach objectivity, contextualization of the analysis and applicability constraints of the Smart Urban Food System. Finally, the study opens a research perspective on the operational Smart Urban Food System modeling, for which this work is the first step.

Keywords: alternative food models / urban food security / sustainable development goals / sustainable urban planning / multicriteria analysis

\section{Introduction}

Dans un contexte de crises économique, écologique et sanitaire, l'enjeu de sécurité alimentaire urbaine devient vital, notamment avec l'accroissement de la population urbaine estimée à plus de la moitié de la population mondiale (Clos, 2018). Nous définissons la sécurité alimentaire urbaine comme la capacité de satisfaire les besoins alimentaires de base d'une population urbaine donnée grâce à un aménagement spatiofonctionnel spécifique (réseaux, équipements, infrastructures, institutions, moyens) qui rend accessible l'alimentation en ville et relève d'une politique de développement durable du territoire. Actuellement, la satisfaction de ces besoins passe soit par l'augmentation de la productivité agricole et la lutte contre le gaspillage alimentaire et/ou par l'importation alimentaire. Or, si la première solution est entravée par les effets du changement climatique (érosion des terres et dégradation des écosystèmes) et les grandes distances dans la chaîne productive, la seconde ne peut être soutenable à cause des crises financières mondiales. Fragilisées déjà par la crise alimentaire de 2008 qui avait impacté négativement les prix des produits agricoles, les villes, notamment celles du Sud, revivent une seconde crise liée à la pandémie de Covid-19 et la fermeture des frontières démontrant, encore une fois, la faiblesse du système agro-industriel mondial et faisant ainsi réémerger le concept de souveraineté alimentaire locale. Cette dernière désigne le droit des pays ou des villes à définir leurs politiques alimentaires sans dépendance de l'extérieur (Lazereg et al., 2020) en décidant de leur propre système productif afin d'assurer des aliments accessibles, nutritifs et culturellement adéquats (Charlier et Warnotte, 2007). Cette situation met en évidence la nécessité de faire évoluer les systèmes alimentaires à travers leur relocalisation, diversification, organisation et intégration (Loconto, 2020). Nous entendons par système alimentaire, l'ensemble organisé d'activités, de produits, de moyens, d'infrastructures et d'acteurs impliqués dans la sécurisation alimentaire à une échelle territoriale donnée (commune ou intercommunalité, ville, métropole, région, pays) et dont l'efficacité relève du management territorial par les collectivités correspondantes.

Le phénomène de relocalisation du système alimentaire existe déjà depuis les années 1990 à travers une nébuleuse de pratiques et d'acteurs se réclamant en rupture avec le système agroalimentaire conventionnel (Deverre et Lamine, 2010). Il a fait naître des modèles alimentaires alternatifs en réponse au risque d'insécurité alimentaire locale. Les modèles alimentaires alternatifs se basent sur le concept des circuits courts, qui ont l'avantage de dynamiser l'économie locale, de réduire les émissions de gaz à effet de serre et l'empreinte écologique et de valoriser la biodiversité locale (Kmec, 2016). Ils compensent ainsi les déficiences écologiques, économiques et sociales causées par le modèle agro-industriel mondial. Sur le plan théorique, la littérature scientifique fait ressortir principalement trois modèles alimentaires alternatifs: le système alimentaire territorialisé (SAT); le City-Region Food System (CRFSSystème alimentaire ville-région) et le système alimentaire urbain intelligent (SAUI). Si les deux premiers modèles sont largement connus et diffusés internationalement, le SAUI est, selon nous, un modèle encore en émergence, grâce à des projets expérimentaux, et n'est pas encore reconnu en tant que modèle à part entière.

La littérature relative à ces modèles met en évidence leur rôle dans l'amélioration de la sécurité alimentaire et le développement local. Cependant, très peu de travaux - voire aucun - se sont penchés sur leur analyse critique au prisme de leur performance dans l'intégration des objectifs de développement durable (ODD) de 1'agenda 2030, qui représente la feuille de route pour des systèmes résilients. De plus, l'approche spatiale par l'aménagement urbain durable demeure quasiment absente dans les quelques travaux d'analyse existants. Il s'agit d'un processus continu, itératif, participatif, holistique et transversal de planification, programmation, conception, réalisation et suivi de l'organisation spatio-fonctionnelle d'un projet alimentaire selon des objectifs préalablement ciblés (optimisation fonctionnelle, santé publique, développement économique local, accessibilité alimentaire, etc.).

C'est pour contribuer à combler ce manque que ce travail propose une évaluation théorique de la performance des modèles alimentaires alternatifs dans l'intégration des ODD par l'aménagement urbain durable (AUD). La première partie de l'article présente une définition des SAT, CRFS et SAUI sur le plan spatio-fonctionnel et selon les quatre domaines de l'écosystème urbain. La deuxième partie présente le cadre méthodologique de l'évaluation par une analyse multicritère choisie après l'identification des méthodes d'analyse existantes. La troisième partie présente et discute les résultats avant de conclure par la discussion des apports théoriques et méthodologiques de ce travail et les perspectives de recherche qu'il ouvre.

\section{Définition des différents modèles alimentaires alternatifs}

Sur la base d'une revue de littérature, nous définissons, dans ce qui suit, les trois modèles alimentaires alternatifs retenus pour l'analyse (Tab. 1). En plus des définitions, des exemples concrets de ces modèles sont donnés dans l'Annexe I disponible en matériel supplémentaire. 


\subsection{Système alimentaire territorialisé (SAT)}

Le modèle territorialisé (SAT) est né en croisant trois concepts : territoire, filière agroalimentaire et résilience. Les travaux de Rastoin (2016) sont pionniers dans la conceptualisation de ce modèle, décrit comme un «ensemble de filières agroalimentaires répondant aux critères du développement durable, localisées dans un espace géographique de dimension régionale et coordonnées par une gouvernance territoriale » (Rastoin, 2016). Le SAT vise une bonne gestion des ressources naturelles à l'échelle régionale tout en limitant l'impact sur l'environnement. Pour cela, il se base sur une double proximité spatiale et fonctionnelle. La première rapproche les filières productives (végétales, animales et forestières) selon les préceptes de l'agro-écologie, tandis que la seconde rapproche l'agriculture et les industries agroalimentaires. Ainsi, des réseaux alimentaires locaux se forment et mutualisent des ressources locales dans le cadre d'un bassin de consommation proche de 1 à 5 millions d'habitants, se développant sur des territoires régionaux, urbains et ruraux.

Le développement d'un SAT est généralement lié à la présence de ressources naturelles et paysagères particulières ainsi qu'à un savoir-faire local. Leur combinaison engendre de nouvelles formes de production visant à valoriser le patrimoine naturel et humain local par un processus d'ancrage territorial porté par les acteurs économiques et civils du territoire. L'économie circulaire, l'innovation et l'écologie jouent un rôle important dans un SAT et contribuent à développer la résilience du modèle face au changement climatique (Rastoin et al., 2016).

\subsection{City-Region Food System (CRFS)}

Le City-Region Food System (CRFS) ou système alimentaire ville-région vise à rapprocher l'alimentation des régions urbaines à travers la relocalisation de l'agriculture autour de la ville (Brand et al., 2017). Un CRFS est défini comme «un réseau complexe d'acteurs, de processus et de relations en rapport avec la production, la transformation, la commercialisation et la consommation alimentaires qui existent dans une région géographique donnée comprenant un centre urbain plus ou moins dense et son arrière-pays périurbain et rural» (Blay-Palmer et al., 2018). Cette conception est basée sur les flux de personnes, de biens, de ressources et de services écosystémiques entre la ville et son territoire (Tecco et al., 2017). L'un des objectifs d'un CRFS est de réduire l'empreinte écologique de l'alimentation. Ainsi, en plus de rapprocher les bassins productifs des villes, les déchets urbains peuvent être valorisés dans les zones périurbaines pour produire de l'énergie et des nutriments dans une logique de boucles métaboliques (Forster et al., 2015). À travers la création de liens espace urbain/espace péri-urbain/arrièrepays, un CRFS cherche aussi à réduire les inégalités territoriales et la marginalisation des petits producteurs en les intégrant dans des circuits courts qui les relient aux marchés urbains. La proximité de la ville leur permet aussi d'accéder aux ressources et services qu'elle offre, comme le transport, la transformation et les financements (FAO, 2014). Dans ce modèle, l'aire métropolitaine s'érige comme l'échelle idéale pour mettre en place des politiques alimentaires adaptées aux contextes socio-économiques et environnementaux locaux (Forster et al., 2015).

\subsection{Système alimentaire urbain intelligent (SAUI)}

Nous considérons le SAUI comme un modèle alimentaire alternatif émergent, dont les signaux faibles sont les projets expérimentaux d'agriculture urbaine et de symbiose industrielle locale. L'agriculture urbaine et la symbiose industrielle sont vues comme les facteurs de résilience de ce modèle face au changement climatique et aux risques naturels et sanitaires. L'agriculture urbaine est une solution d'avenir qui pourrait améliorer la sécurité alimentaire urbaine, notamment pour les villes vulnérables (Specht et al., 2014). Elle repose sur un système productif hybride qui associe des technologies hard et soft. Les premières sont des technologies de bas rendement et concernent l'agriculture située dans les interstices urbains. Les secondes sont plutôt de haut rendement et intelligentes, dont l'exemple le plus connu est celui des fermes verticales popularisées par Despommier (2010) et qui pourraient répondre au problème de pénurie de terres arables dans les villes denses (Benis et Ferrão, 2017). Les entités productives du SAUI doivent s'intégrer dans un cycle métabolique fermé qui englobe les autres flux de matières urbaines comme les déchets organiques, l'eau et l'énergie. Le métabolisme circulaire permet ainsi d'optimiser le modèle sur les plans économique, environnemental et social (Lange et al., 2017).

Sur le plan spatial, des travaux mêlant recherches et expériences de terrain œuvrent pour intégrer le SAUI dans une nouvelle approche architecturale et urbanistique (Verzone et Dind, 2011). L'aménagement urbain qui en découle doit prendre en charge l'aspect multifonctionnel de l'agriculture urbaine (Aubry et Pourias, 2013) et repenser les intercommunalités urbaines sous l'angle des symbioses industrielles (circuits courts spécialisés, mutualisation des déchets et des équipements, etc.) et des seuils de densités (BerezowskaAzzag et al., 2014).

\section{Méthodologie}

\subsection{Choix de la méthode}

Dans la littérature scientifique, il existe 12 méthodes multicritères d'évaluation de la durabilité agricole que Van Der Werf et Petit (2002) ont caractérisées selon l'objet d'évaluation, l'approche utilisée et la dimension considérée. Parmi elles, nous pouvons citer la méthode par Indicateurs de durabilité des exploitations agricoles-IDEA (Zahm et al., 2019), la méthode par les Indicateurs agro-écologiques (Girardin et Bockstaller, 1997) ou la méthode par analyse du cycle de vie pour l'agriculture (Pradel, 2011). Cependant, ces méthodes ne pouvaient s'appliquer ici pour deux raisons. D'une part, elles évaluent la durabilité de l'agriculture à l'étape de la production, or notre analyse des modèles alimentaires alternatifs se veut systémique et intégrée en prenant en considération à la fois les éléments de durabilité de référence d'un système alimentaire (sécurité alimentaire, résilience au changement climatique, innovation, etc.) et ceux de son intégration spatiale urbaine (planification intégrée, structure spatiale, infrastructures et fonctions, gouvernance participative, etc.). 


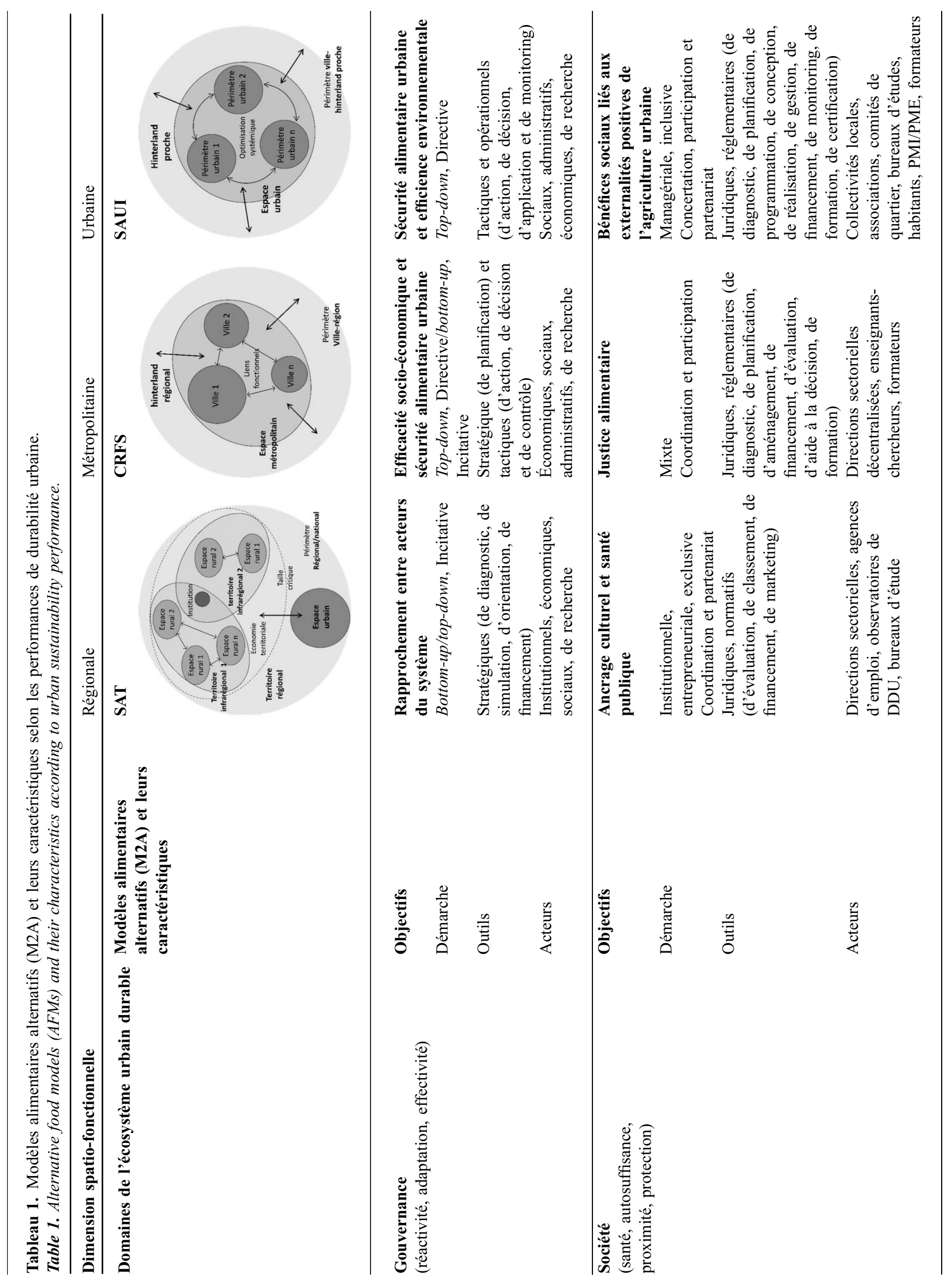

Page 4 de 15 


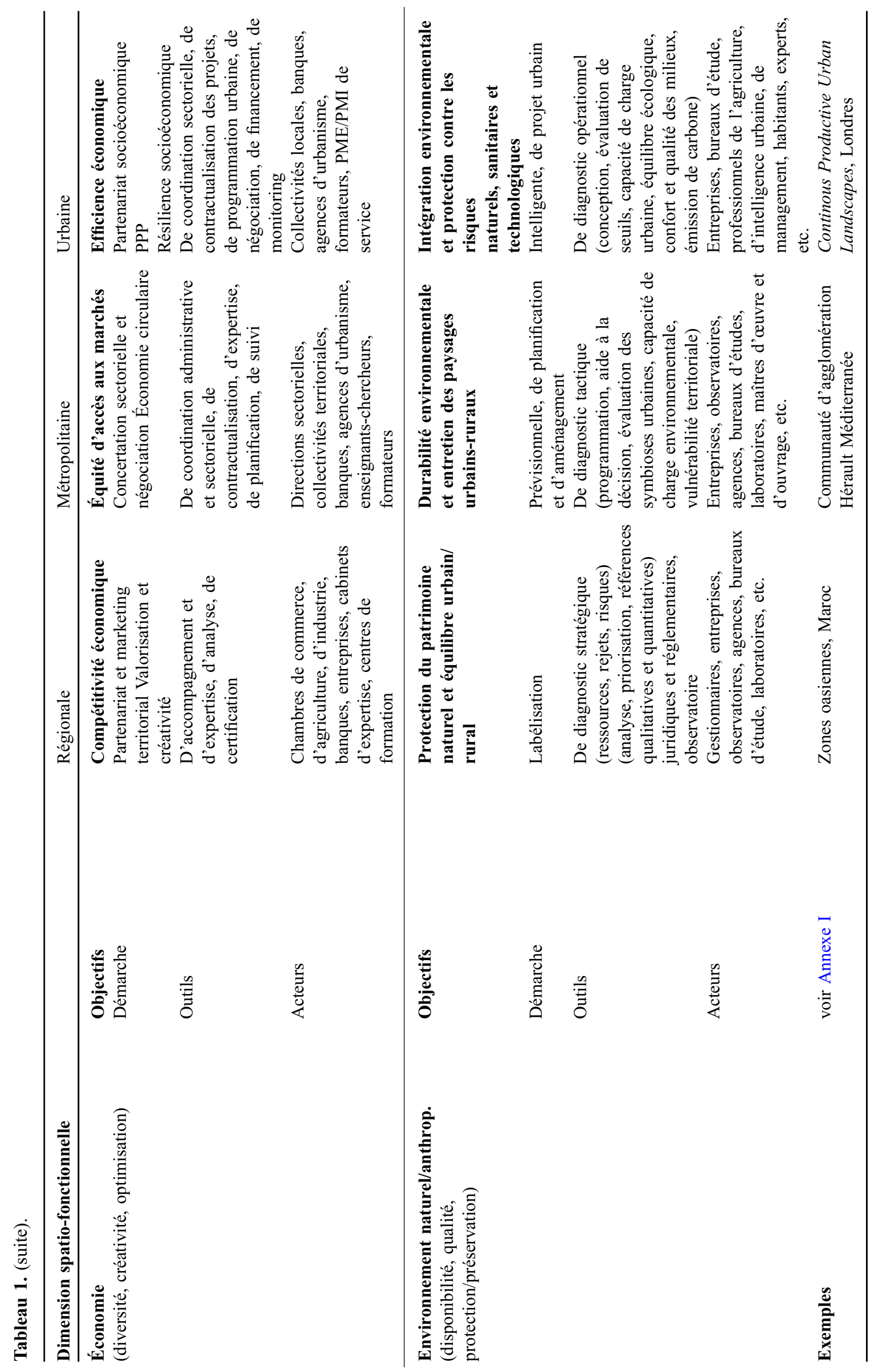

Page 5 de 15 
D'autre part, elles sont non exhaustives dans la mesure où chacune s'intéresse à un domaine particulier de la durabilité qui peut être soit environnemental, économique ou social (Halimi, 2014). En revanche, analyser la durabilité urbaine d'un modèle alimentaire nécessite de le considérer selon une approche exhaustive qui tienne compte à la fois de tous les domaines de durabilité écosystémique. C'est pourquoi nous avons établi notre propre méthode d'analyse multicritère qui se base sur le croisement de deux approches évaluatives complémentaires déclinées en cibles d'analyse. Du point de vue théorique, sans prétendre être exhaustive, notre méthode se veut plus holistique que les méthodes existantes d'évaluation des performances de durabilité des systèmes agricoles.

\subsection{Choix des approches évaluatives et des cibles d'analyse}

\subsubsection{Approche normative par les objectifs de développement durable (ODD)}

En vue d'analyser la durabilité d'un modèle alimentaire, il est important d'adopter d'abord une approche normative de référence. Une telle approche a pour rôle d'indiquer la direction à prendre pour aller vers la concrétisation des visions et objectifs de durabilité dans les modèles (Sala et al., 2015). Dans notre analyse, cette approche normative est incarnée par les 17 objectifs de développement durable (ODD) de l'agenda 2030 défini par l'ONU. Ils constituent un référentiel théorique et descriptif qui implique les cibles et actions à mettre en œuvre pour que le modèle alimentaire soit durable (Zahm et al., 2019). Ce choix s'explique d'abord par la nature transversale, systémique et intégrée des ODD, dont beaucoup touchent directement ou indirectement à la thématique alimentaire. D'un autre côté, il existe un consensus mondial sur les objectifs de l'ONU, qui leur confère un caractère légitime en tant que cadre d'analyse.

\subsubsection{Approche opérationnelle par l'aménagement urbain durable (AUD)}

L'aménagement urbain durable (AUD) est le moyen d'intégration et de mise en œuvre de la durabilité urbaine. Il implique des politiques de planification urbaine et des outils stratégiques et opérationnels comme l'Agenda 21, un Plan climat-air-énergie territorial ou un Projet alimentaire territorial (Jégou et al., 2012). L'AUD est alors un outil qui permet, de manière générale, de rendre opérationnels des objectifs de développement durable et, de manière spécifique, d'intégrer le système alimentaire en milieu urbain. Dans le cadre de notre analyse, nous considérons l'AUD comme une approche évaluative opérationnelle qui permet de vérifier le degré d'intégration urbaine des ODD dans les modèles alimentaires alternatifs étudiés et de dépasser l'aspect normatif de l'analyse en l'inscrivant dans une approche théorique spatiale et opérationnelle. Nous considérons que les deux démarches évaluatives choisies sont complémentaires.

\subsubsection{Choix des domaines et cibles d'évaluation concernés par l'analyse}

La définition des domaines et cibles d'évaluation s'est faite à travers un travail bibliographique relatif aux ODD et à l'AUD, selon le principe du respect des critères suivants :
- constat d'un lien direct et fort avec la durabilité urbaine d'un système alimentaire ;

- possibilité de vérification des cibles dans chaque modèle grâce à l'existence d'une revue de littérature descriptive.

Les principales références bibliographiques sont le site des Nations Unies (2020) et le Cerema, 21 C (2019) pour les ODD; le Référentiel européen des villes et territoires durables (RFSC: http://rfsc.eu/); Boutefeu (2006) pour la méthode RST02; l'Agence régionale pour l'environnement de MidiPyrénées en France pour les indicateurs de développement urbain durable; Charlot-Valdieu et Outrequin (2004) pour la méthode $\mathrm{HQE}^{2} \mathrm{R}$; et Srir (2013) pour les référentiels de l'aménagement urbain durable. Ces références nous ont permis d'identifier 4 domaines de l'écosystème urbain (gouvernance, société, économie, environnement) et 19 cibles d'évaluation pour l'AUD (Tab. 2), ainsi que 7 domaines et 24 cibles pour les ODD, notamment: ODD 2 « Sécurité alimentaire et agriculture durable»; ODD 8 « Travail décent et croissance économique»; ODD 9 «Industrie, innovation et infrastructures»; ODD 11 «Villes et communautés durables »; ODD 12 «Consommation et production responsables»; ODD 13 «Lutte contre le changement climatique » et ODD 15 «Vie terrestre» (Tab. 3).

En raison du caractère systémique et transversal des ODD, certains d'entre eux n'ont pas été retenus pour l'analyse afin d'éviter des redondances. A titre d'exemple, parmi les cibles de l'ODD 1 «réduction de la pauvreté », figure l'accès à la nourriture, l'amélioration de la résilience des villes et l'adaptation au changement climatique qui sont déjà prises en charge respectivement par les ODD 2, 11 et 13. Un autre exemple est celui de l'ODD 6 « eau propre et assainissement » dont les cibles sont liées à celles de 1'ODD 2 par les pratiques agricoles résilientes (capacité d'adaptation aux sécheresses et inondations), de l'ODD 12 par la gestion durable et rationnelle des ressources naturelles dont l'eau et de l'ODD 15 à travers la préservation des écosystèmes terrestres.

\subsection{Structure de la matrice d'analyse multicritère et méthode d'évaluation}

La figure 1 montre le canevas de la matrice servant à l'analyse multicritère d'évaluation de la performance des modèles alimentaires alternatifs dans la prise en charge des ODD par l'aménagement urbain durable, avec les 24 cibles des ODD disposées en colonnes et les 19 cibles de l'AUD en lignes. La matrice est renseignée séparément pour chaque modèle alimentaire alternatif et interroge les relations entre les cibles selon: (1) l'appropriation des ODD par les 4 domaines de l'écosystème urbain (lecture horizontale des relations) et (2) l'adéquation des 19 cibles de l'AUD aux 7 ODD (lecture verticale des relations). En considérant les états de savoir issus des lectures et exemples étudiés, une relation correspond à un croisement vérifié d'une cible ODD avec une cible AUD, selon la question préalablement définie: «Est-ce que la cible A de l'ODD $\alpha$ est prise en charge par la cible B du domaine $\beta$ de l'AUD ? ». Le signe [X] renseigné dans la case de croisement indique l'existence d'une relation. Les grilles ont été remplies de manière séparée par les auteurs de l'article en leur qualité d'experts dans le domaine de l'aménagement urbain durable et 
Tableau 2. Dix-neuf cibles de l'approche opérationnelle par l'aménagement urbain durable (AUD). Table 2. Nineteen targets of the operational approach by Sustainable Urban Planning (SUP).

\begin{tabular}{llll}
\hline Cible & Description en rapport avec la sécurité alimentaire urbaine & D'après les sources \\
\hline $\begin{array}{lll}\text { 1. Consultation et participation des } \\
\text { différents acteurs }\end{array}$ & $\begin{array}{l}\text { Mobilisation d'acteurs (communes, intercommunalités, } \\
\text { agriculteurs, chambres consulaires, transformateurs, }\end{array}$ & Darrot et al., 2019
\end{tabular}

différents acteurs

\section{Coopération intercommunale}

\section{Outils d'aide à la décision}

4. Outils d'évaluation et de suivi de l'état de l'environnement

\section{Accessibilité urbaine à l'alimentation}

6. Préservation de la santé

\begin{abstract}
7. Accessibilité aux services de proximité
\end{abstract}

8. Multifonctionnalité de l'agriculture

9. Équité et inclusion sociale

10. Création d'éco-emploi

11. Valeur créative

12. Diversification des activités

13. Optimisation fonctionnelle du système

14. Équilibre d'aménagement espaces naturels/espaces anthropiques agriculteurs, chambres consulaires, transformateurs, consommateurs, etc.) dans l'élaboration de politiques publiques alimentaires locales.

Processus par lequel les élus élaborent et révisent leurs actions de planification alimentaire dans une démarche intercommunale limitant les dynamiques concurrentielles entre territoires.

Outils d'encadrement des politiques alimentaires, de diagnostic, de mise en œuvre de planification alimentaire, de monitoring, d'information et de concertation.

Permettent de mesurer et analyser les effets d'une politique publique ou d'un projet structurant alimentaire sur l'environnement afin de prévenir des conséquences dommageables sur ce dernier.

Stabilité d'accès à une nourriture adéquate en ville. Cela couvre l'accès physique et économique des ménages à la nourriture.

Liée à la qualité sanitaire des aliments et les modes de production et favorise des alternatives durable comme l'agro-écologie qui satisfait des objectifs de préservation de l'environnement.

Accessibilité des acteurs du système alimentaire à des services marchands ou non marchands (administrations publiques, commerces, transport, activités de gestion, communication, etc.) dont la proximité est liée à leurs conditions de déplacement et aux modalités de desserte de ces services.

Agriculture qui intervient dans les différentes sphères dont l'aménagement urbain, l'environnement, l'économie, la sécurité alimentaire, la santé, le loisir et l'éducation. Principes de la justice alimentaire consistant à faire participer les citoyens marginalisés à la production de leurs aliments dans l'objectif de leur offrir un accès digne à l'alimentation.

Fonctions nouvelles ou déjà existantes occupées dans le but de protéger l'environnement, issues de mutation et/ou de création de certains métiers dans le cadre d'une économie verte.

Implique la création de nouveaux produits agricoles, de nouvelles utilisations ou de nouveaux procédés touchant toute la chaîne de valeur, de la production à la consommation.

Basée sur des innovations qui profitent notamment aux petites entreprises pour diversifier les activités, créer de nouveaux débouchés commerciaux et améliorer les revenus.

Basée sur les échanges de matières, d'eau et d'énergie s'appuyant sur la proximité et l'organisation en réseau en vue d'améliorer l'efficience des organisations logistiques. Fait appel à des dispositifs innovants comme les bioraffineries environnementales.

Permet un arbitrage juste entre espaces naturels et écosystèmes d'un côté et espaces urbanisés par le logement, l'industrie, les transports, etc. de l'autre, en tenant compte de la biocapacité et en faisant appel à un mode de planification concerté entre les différentes parties.
Loubet, 2012

Berezowska-Azzag, 2011

ADEME, 2020

FAO

Angeon et Barraud, 2019

Pépin, 2000

Wegmuller et Duchemin, 2010

Beischer et Corbett, 2016

Arib, 2014

FAO, 1996

FAO, 2018

Guilbert et Redlingshöfer, 2018

Auteurs 
Tableau 2. (suite).

\begin{tabular}{|c|c|c|}
\hline Cible & Description en rapport avec la sécurité alimentaire urbaine & D'après les sources \\
\hline 16. Densité urbaine optimale & $\begin{array}{l}\text { Densité qui répond au juste milieu entre densité voulue } \\
\text { pour atteindre les objectifs locaux de durabilité et le degré } \\
\text { d'acceptabilité d'une densité physique et sociale donnée. } \\
\text { Elle obéit au principe de «charge admissible» qui permet } \\
\text { d'optimiser la consommation des ressources et des coûts. }\end{array}$ & $\begin{array}{l}\text { Amphoux et al., 1999; } \\
\text { Thalmann et Doyle, } 2013\end{array}$ \\
\hline 17. Mobilité durable & $\begin{array}{l}\text { Existe lorsque les distances géographiques entre producteur } \\
\text { et consommateur sont limitées, induisant des circuits courts } \\
\text { de proximité qui constituent des bassins de consommation } \\
\text { proches. }\end{array}$ & Aubry et Chiffoleau, 2009 \\
\hline $\begin{array}{l}\text { 19. Résilience aux risques naturels, } \\
\text { sanitaires et technologiques }\end{array}$ & $\begin{array}{l}\text { Un système alimentaire est résilient lorsqu'il est diversifié } \\
\text { (agriculture urbaine, périurbaine et rurale); distribué } \\
\text { (renforcement des liens ville-territoire); naturel (meilleure } \\
\text { gestion des ressources naturelles); innovant (technologies, } \\
\text { métabolisme urbain circulaire); social (emplois, choix } \\
\text { alimentaires, etc.) et inclusif (gouvernance participative). }\end{array}$ & Dansero et al., 2016 \\
\hline
\end{tabular}

connaisseurs des particularités de chaque modèle (Annexes II, III et IV disponibles en matériel supplémentaire). À l'issue de l'exercice, la comparaison des matrices a fait ressortir pour chaque modèle des croisements litigieux, discutés ensuite et résolus avec l'appui d'experts externes consultés en cas de doute.

Dans la matrice, les croisements ont été interprétés par des scores. Le score obtenu par chaque cible d'analyse est égal à la somme des signes [X] par colonne (score d'adéquation des cibles de l'AUD aux ODD) et par ligne (scores d'appropriation des cibles ODD par les 4 domaines de l'écosystème urbain). Sur cette base, trois taux ont été calculés pour chaque modèle alimentaire alternatif: un taux d'adéquation des 4 domaines de l'AUD avec les ODD ciblés (formule (1)), un taux d'appropriation des 7 ODD par les domaines de l'écosystème urbain (formule (2)) et un taux de performance urbaine globale par modèle (formule (3)).

Taux de prise en charge des ODD par l'aménagement urbain durable $\left(\mathrm{T}_{A U D}\right)$ :

$$
\begin{aligned}
T_{A U D} & =\frac{\sum \text { cibles AUD adéquates aux cibles d'un ODD donné }}{\sum_{\text {max de croisements possibles pour cet ODD }}} \\
& \times 100 \% .
\end{aligned}
$$

Taux d'appropriation des ODD par domaine de l'écosystème urbain $\left(\mathrm{T}_{O D D}\right)$ :

$$
\begin{aligned}
& T_{O D D}= \\
& \frac{\sum \text { cibles ODD intégrées dans un domaine donné de l'AUD }}{\quad \sum_{\max } \text { de croisements possibles pour ce domaine }} \\
& \quad \times 100 \% .
\end{aligned}
$$

Taux de performance urbaine globale pour le modèle donné ( $\left.\mathrm{T}_{\text {Modèles Alimentaires Alternatifs }}\right)$ :

$$
\begin{array}{r}
T_{M 2 A}=\text { Moyenne arithmétique des } T_{O D D} \text { par } \\
\text { domaine de l'écosystème urbain. }
\end{array}
$$

Il convient de préciser que :

- le seuil de performance souhaitable du modèle est fixé au $2 / 3$ du taux donné ;

- aucune pondération n'est appliquée dans cette méthode, qui considère tous les ODD et tous les AUD également importants dans la performance théorique du modèle.

Cependant, la contextualisation de l'évaluation pourrait changer les résultats en fonction des paramètres locaux qui seront discutés plus bas. Les contraintes liées aux enjeux et aux politiques locales de développement durable seraient alors susceptibles de modifier le poids des ODD et de leurs cibles, alors que les contraintes d'ordre juridique, foncier, financier, 
Tableau 3. Vingt-quatre cibles de l'approche normative par les objectifs de développement durable (ODD).

Table 3. Twenty-four targets of the normative approach by Sustainable Development Goals (SDG).

Cible $\quad \begin{aligned} & \text { Description en rapport avec la sécurité alimentaire D'après les sources } \\ & \text { urbaine }\end{aligned}$
urbaine

1. Accès durable à une alimentation saine et suffisante

\section{Augmentation de la productivité agricole}

3. Résilience du système productif

4. Recherche \& Développement

5. Diversification et modernisation technologique

6. Créativité et innovation des entreprises et création d'emplois

7. Efficience de la production et de la consommation

8. Mise en valeur des produits locaux

9. Infrastructures de qualité, fiables et résilientes

10. Technologies et procédés propres

11. Emploi des technologies de l'information et de communication

12. Planification urbaine durable
Relève de la sécurité alimentaire et est satisfait lorsque chacun a accès à tout instant, physiquement et économiquement, à une alimentation adéquate lui permettant de satisfaire ses besoins énergétiques pour mener une vie saine et active.

Permet d'assurer la sécurité alimentaire et augmenter les revenus des petits producteurs. Cela passe par l'accès aux ressources productives, au savoir, aux services financiers, au marché, etc. Se base sur des pratiques permettant d'accroître la production, la préservation des écosystèmes, le renforcement des capacités et l'adaptation aux effets du changement climatique.

Mise au point de services de recherche et de vulgarisation agricole pour aider à mettre en place des technologies innovantes en faveur du renforcement des capacités productives et de la durabilité.

Recours aux outils numériques notamment pour améliorer la collecte des données dans le système productif, accroître les rendements, optimiser les intrants, etc.

Fait appel aux incubateurs, aux parcs technologiques, etc., pour améliorer la puissance d'innovation des entreprises qui se base sur la diffusion des technologies pour mettre en œuvre des produits, des processus ou des modes d'organisation nouveaux.

Passe par la limitation de la surproduction d'un côté et l'éducation nutritionnelle de l'autre pour l'adaptation du régime alimentaire et la limitation des impacts écologiques et la consommation énergétique.

Création de nouveaux produits, de nouvelles utilisations ou de nouvelles présentations à partir de produits locaux bruts selon un savoir-faire local.

Condition d'un système productif intégré qui se base sur l'existence de routes, marchés, transport, télécommunications, capacité de stockage, technologies modernes, etc. permettant de maintenir un certain niveau de fonctionnement face au changement climatique.

Technologies vertes qui rendent le système agricole moins tributaire des intrants à fort contenu énergétique dans le but de préserver

l'environnement.

Notamment dans l'agriculture intelligente. Permet un recueil de données en temps réel dans le système, le développement de solutions adaptées et la prise de décision autonome. Aide à la mise au point de systèmes d'alerte en cas de risques de catastrophe.

Favorise une répartition cohérente des activités (agricoles et non agricoles) sur un territoire donné
FAO, 2006

UN

UN

Nations Unies, 2020

Nations Unies, 2020; FAO

FAO, 2018

FAO, 1996

FAO, 2018

FAO

FAO

Bousmaha et Boulkaibet, 2019 
Tableau 3. (suite).

Cible $\quad \begin{aligned} & \text { Description en rapport avec la sécurité alimentaire D'après les sources } \\ & \text { urbaine }\end{aligned}$
urbaine

13. Protection et préservation du patrimoine naturel

14. Gestion efficace des déchets

15. Liens fonctionnels zones urbaines et zones périurbaines rurales

16. Gestion durable des ressources naturelles 17. Réduction des pertes et des déchets dans la chaîne de production

18. Contrôle des impacts sur le développement durable

19. Résilience et capacités d'adaptation aux aléas climatiques

20. Politiques, stratégies et planification adaptées au changement climatique

21. Capacités individuelles, collective et institutionnelles d'adaptation

22. Préservation et exploitation durable des écosystèmes

23. Mesures énergétiques en faveur de l'environnement naturel

24. Planification intégrée en faveur de la protection des écosystèmes et assure une gestion rationnelle des ressources comme les terres agricoles selon un cadre réglementaire.

Protection du patrimoine naturel aux

Auteurs caractéristiques paysagères et écologiques particulières par les plans de développement et d'aménagement pour développer une activité productive et/ou touristique locale.

Dépend du principe d'économie circulaire comme alternative à l'économie traditionnelle linéaire (production-utilisation-élimination) pour optimiser les ressources naturelles et récupérer les déchets par des sous-acteurs.

Synergies entre les centres urbains et leur hinterland agricole pour permettre aux producteurs marginalisés de placer leurs produits dans les marchés et avoir accès aux infrastructures situés en milieu urbain.

Liée aux cibles 12,13 et 14 .

Liée aux cibles 7 et 14 .

Utilise des outils d'aide à la décision pour identifier les impacts potentiels d'un projet alimentaire sur les domaines de développement (social, économique et environnemental) afin d'incorporer des mesures de prévention, d'atténuation et de suivi appropriés.

Lié aux cibles, 3, 9 et 21 .

Nécessite l'intégration du système alimentaire dans les politiques, les stratégies et les plans d'actions locaux, régionaux et nationaux via des processus et des structures institutionnelles opérationnelles.

Basées sur la sensibilisation, l'évaluation

systémique et prévisionnelle des effets du changement climatique, la mise au point de systèmes d'alerte et l'adaptation des pratiques en mettant en place des outils comme les plans climats.

Passe par la mise au point de systèmes d'exploitation durables qui permettent aux écosystèmes de continuer à fournir leurs biens et services de manière durable.

Consistent à accroître la part de l'énergie renouvelable dans le bouquet énergétique employé dans le système productif en développant des infrastructures et technologies d'énergie propre.

Contrairement à la planification conventionnelle, elle se base sur une vision holistique et exhaustive qui prend en charge tous les facteurs impliqués dans le développement des ressources et écosystèmes dans le cadre de l'aménagement urbain ou rural au niveau local.
Auteurs

FAO, 2018

FAO, 2007

UN

FAO 


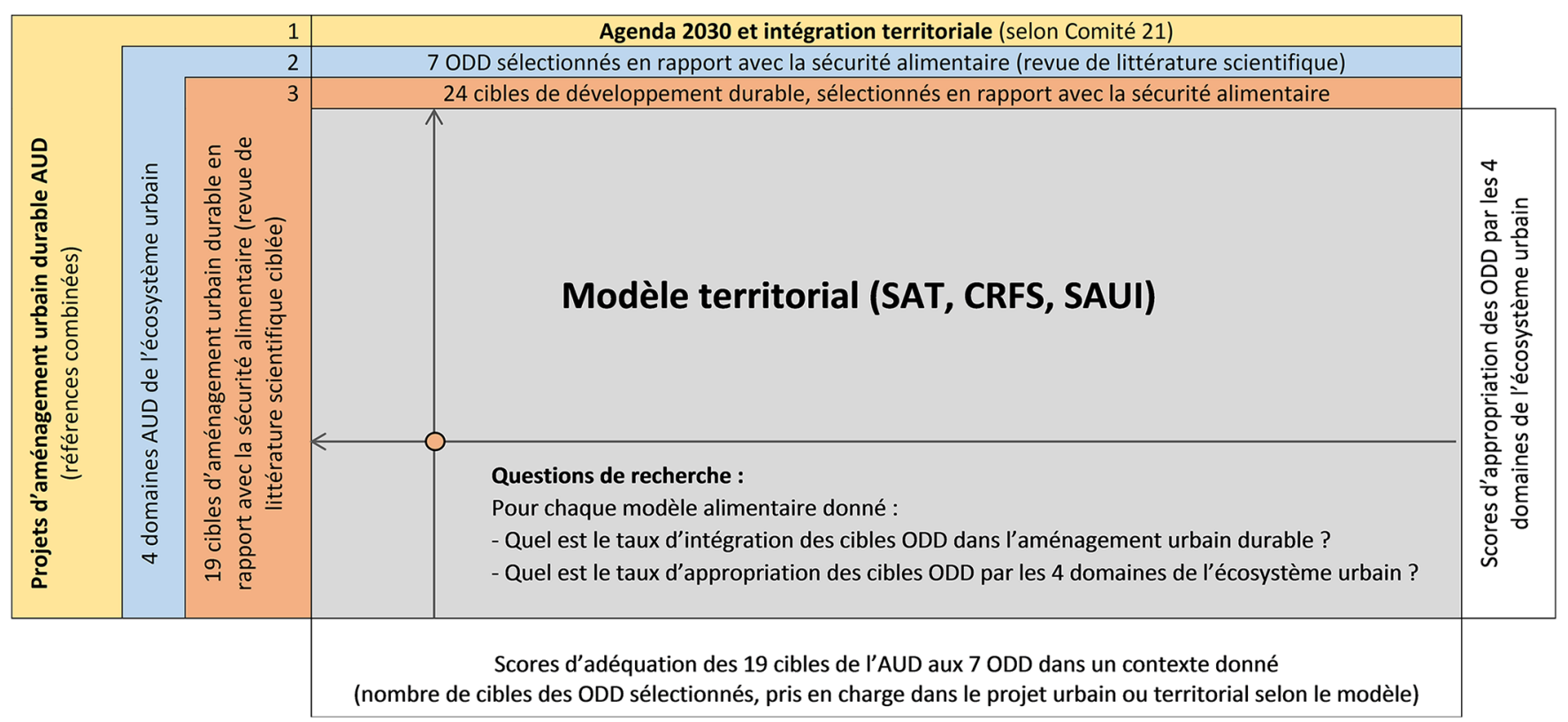

Fig. 1. Canevas de la matrice d'évaluation de la performance des modèles alimentaires alternatifs (M2A) dans la prise en charge des ODD par l'aménagement urbain durable (AUD).

Fig. 1. Matrix framework for assessing the performance of alternative food models (AFMs) in implementing the SDGs through Sustainable Urban Development (SUD).

technique et technologique, pourraient influer sur les capacités locales de prise en charge des ODD et modifier le poids des domaines d'aménagement urbain durable.

\section{Résultats et discussion}

Les résultats des analyses nous ont permis de définir les niveaux de performance de chaque modèle alimentaire alternatif par rapport à la prise en charge des ODD dans l'aménagement urbain durable. Les graphes comparatifs des résultats illustrent les taux d'intégration des 7 ODD ciblés ainsi que les taux d'adéquation des 4 domaines de l'AUD dans chaque modèle (Fig. 2 et 3). On remarque d'abord que le degré de performance des modèles évolue graduellement en fonction du degré de leur proximité urbaine.

En effet, les taux d'intégration des 7 ODD ciblés dans le SAUI se situent au niveau ou au-dessus du seuil minimum moyen d'intégration des ODD. Ce serait alors le modèle le plus performant. À l'opposé du SAUI, le SAT serait le modèle le moins performant avec des taux d'intégration qui se situent en dessous du seuil. Quant au CRFS, ses taux d'intégration sont parfois supérieurs, parfois inférieurs, au seuil défini; il peut être alors considéré comme un modèle de performance moyenne. Nous pouvons expliquer ces résultats par le fait que les villes et les régions urbaines deviennent les échelons adéquats pour la construction de systèmes alimentaires durables (Viljoen et Wiskerke, 2012) pour faire face à la «nouvelle équation alimentaire» (Morgan et Sonnino, 2010) qui inclut le changement climatique, l'urbanisation accélérée et les externalités négatives du modèle agro-industriel dominant. Cette tendance est conjointe à celle de la décentralisation qui offre une marge de manœuvre non

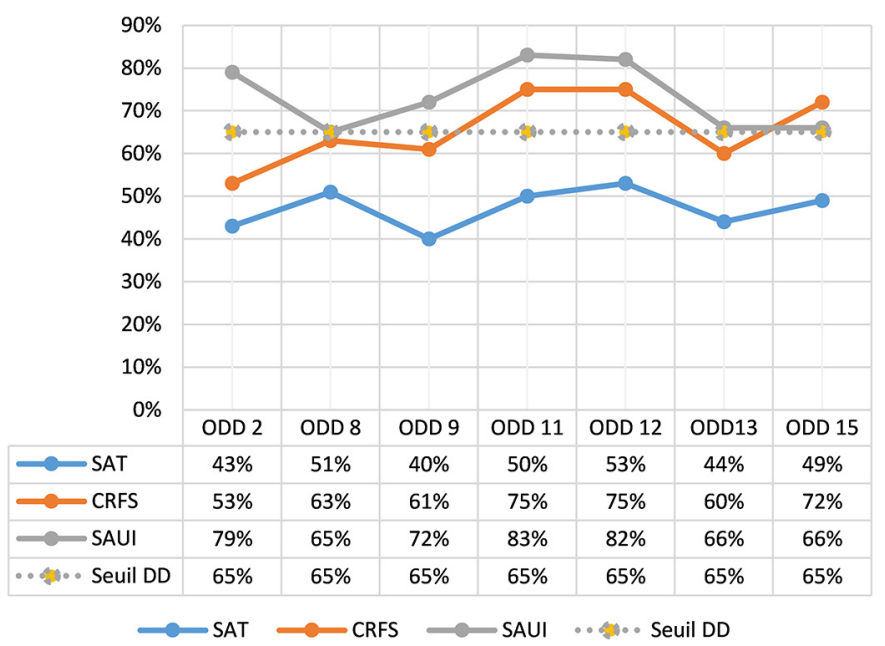

Fig. 2. Taux d'integration des 7 ODD ciblés dans les modèles alimentaires alternatifs (M2A).

Fig. 2. Integration rates of the seven targeted SDGs in alternative food models (AFMs).

négligeable aux collectivités locales pour intégrer la question alimentaire dans les stratégies de développement local et de planification urbaine.

Cependant, malgré les différences de performance, les 3 modèles ont en commun un même objectif fondamental qui est celui de la consommation et la production responsables (ODD 12). Ce dernier affiche les taux d'intégration les plus importants dans les 3 modèles ( $82 \%$ dans le SAUI, $75 \%$ dans le CRFS et $53 \%$ dans le SAT). Cela s'explique par le fait que les ressources naturelles sont considérées comme vitales pour des systèmes alimentaires durables comme le SAT, le CRFS et 


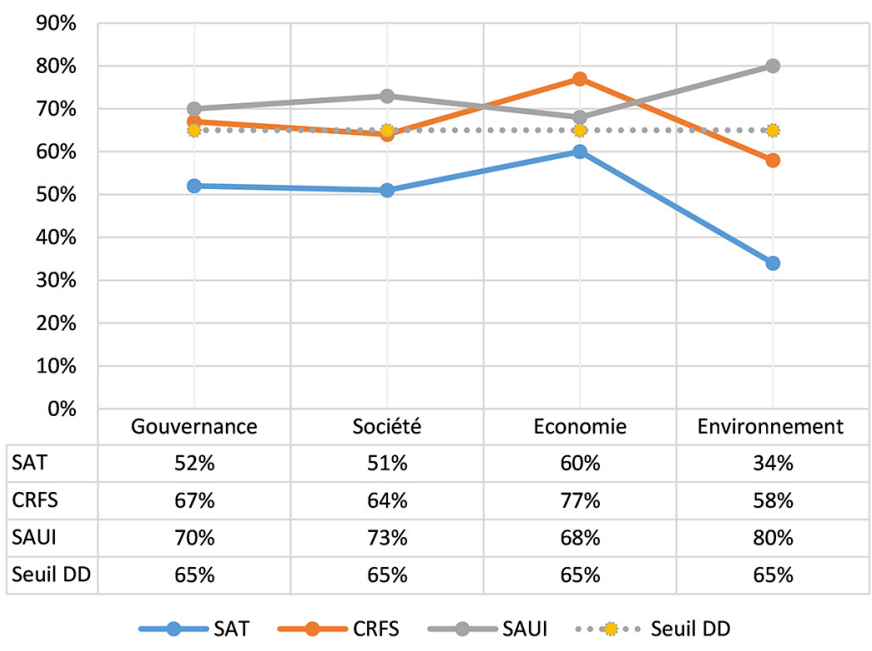

Fig. 3. Taux d'adéquation des 4 domaines de l'aménagement urbain durable (AUD) avec les ODD ciblés dans les modèles alimentaires alternatifs (M2A).

Fig. 3. Adequacy rates of the four Sustainable Urban Planning (SUP) domains with the targeted SDGs in the alternative food models (AFMs).

le SAUI qui prônent une diversité de l'alimentation la plus forte possible, soutenue par la diversité biologique locale, support des modèles durables contrairement au modèle agroindustriel mondialisé. Aussi, une complémentarité des objectifs entre ces modèles pourrait-elle être envisagée, vu leurs profils d'échelle et d'objectifs différents. En effet, la comparaison des taux d'adéquation des 4 domaines de l'AUD avec les ODD ciblés dans les 3 modèles analysés, fait ressortir un profil économique pour les le SAT et le CRFS en rapport avec leur taux d'adéquation le plus élevé, à savoir, $60 \%$ et $77 \%$ respectivement. Quant au SAUI, c'est plutôt un profil socio-environnemental qui ressort avec des taux d'adéquation de $73 \%$ dans le domaine social et $80 \%$ dans celui environnemental.

La sécurité alimentaire urbaine pourrait, d'après les résultats de cette évaluation, être améliorée fondamentalement grâce aux modèles urbains que sont le SAUI et le CRFS. En plus de l'intégration de l'ODD 12 (consommation et production responsables), les deux modèles intègrent aussi de manière importante l'ODD 11 relatif aux villes et communautés durables $(82 \%$ dans le SAUI, $75 \%$ dans le CRFS). En effet, la durabilité des villes de manière générale et du système alimentaire de manière spécifique, est le fruit des efforts des collectivités en termes de renforcement de l'urbanisation inclusive (liens fonctionnels entre zones urbaines et zones périurbaines) et durable (protection du patrimoine naturel et gestion efficace des déchets) dans le cadre d'une planification urbaine holistique qui prend en charge les circuits courts alimentaires, les services de proximité, l'agriculture urbaine et péri-urbaine, les densités optimales et le métabolisme circulaire. Par ailleurs, avec une importance moindre et presque similaire, le SAUI et le CRFS intègrent l'ODD 8 (travail décent et croissance économique), dont les taux se situent autour du seuil minimum moyen d'intégration ( $65 \%$ et $63 \%$ respectivement). Le développement économique à l'échelle urbaine est inhérent à la créativité des entreprises locales qui participent à l'efficience de la chaîne productive et à la création de valeurs ajoutées par des technologies et procédés innovants mis en œuvre dans toutes les actions de programmation et de conception d'aménagement.

La préservation durable des écosystèmes (ODD 15) est, toutefois, plus intégrée dans le CRFS que dans le SAUI (72\% et $66 \%$ respectivement). C'est effectivement à l'échelle métropolitaine que se situe l'enjeu de complémentarité fonctionnelle et de conciliation des objectifs économiques et environnementaux d'une région urbaine. Les collectivités doivent assurer la coordination de l'aménagement urbain par le biais de plans métropolitains, cruciaux pour l'optimisation des infrastructures et des équipements urbains servant aussi bien à l'industrie, à l'habitat et à l'agriculture en vue de préserver l'équilibre des écosystèmes. Du point de vue environnemental, ces plans doivent tenir compte de la préservation des grands écosystèmes et de la biodiversité ainsi que de la protection du système alimentaire des catastrophes naturelles (inondations et érosions).

Le modèle du SAUI affiche une meilleure capacité à améliorer la sécurité alimentaire urbaine que le modèle villerégion à travers un taux d'intégration de l'ODD 2 «sécurité alimentaire et agriculture durable » nettement supérieur $(79 \%$ et $53 \%$ respectivement). La sécurité alimentaire à l'échelle de la ville est confortée par l'essor de «l'urbanisme agricole» (Boucher, 2009) qui prône l'intégration de l'agriculture à la croissance urbaine. Pour ce faire, la planification urbaine vise à créer un environnement qui appuie l'agriculture urbaine (soft et/ou hard) par le développement d'équipements et d'infrastructures, souvent innovants, ce qui explique le taux d'intégration important de l'ODD 9 «industries, innovations et infrastructures » dans le SAUI $(72 \%)$.

L'environnement ainsi créé doit afficher une densité urbaine optimale pour respecter la capacité de charge urbaine, permettre des déplacements durables et un fonctionnement optimisé des équipements. L'autonomie alimentaire locale et l'augmentation des capacités productives urbaines constituent des enjeux mobilisateurs pour lesquels tout un appareil scientifique et technique s'érige afin de créer des systèmes alimentaires urbains intelligents, collaboratifs, sobres et robustes. L'innovation urbaine réside aussi dans la construction de nouveaux agencements politiques locaux et de nouveaux modes de gouvernance urbaine participative et prospective, d'où le fort taux d'adéquation du domaine de gouvernance aux ODD ciblés dans le SAUI ( $70 \%$ contre $67 \%$ et $52 \%$ pour le CRFS et le SAT respectivement).

Bien que les résultats obtenus confortent notre hypothèse de départ, l'évaluation présente deux limites principales. La première est que l'évaluation a été effectuée sur les modèles théoriques, elle reste donc abstraite. Dans le cas d'un contexte donné, l'évaluation devrait intégrer la pondération des ODD selon l'importance des enjeux locaux qui diffère d'un contexte à un autre (changement climatique, crise écologique, crise énergétique, degré d'urbanisation, efficacité de la gouvernance, faiblesse d'intégration scientifique et technologique, compétences humaines, etc.). De ce fait, la formule de calcul des taux d'intégration des ODD devrait intégrer des coefficients tels que celui de la capacité de gestion ou d'adaptation des collectivités locales concernées ou la disponibilité des moyens d'action. Dès lors, ces coefficients 


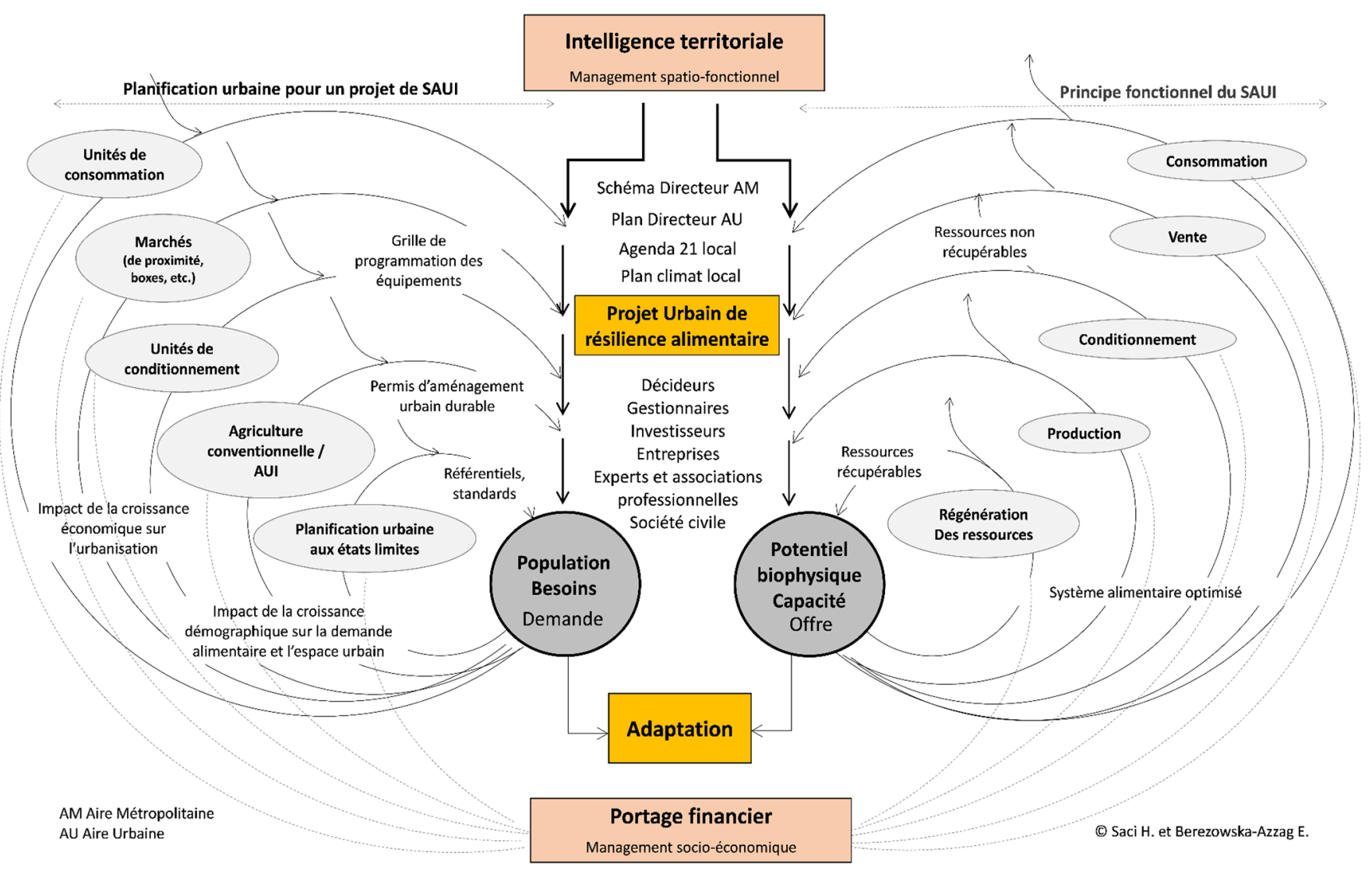

Fig. 4. Modèle théorique du système alimentaire urbain intelligent (SAUI).

Fig. 4. Theoretical model of Smart Urban Food System (SUFS).

pourraient augmenter ou diminuer le taux dans un domaine donné de manière proportionnelle ou inversement proportionnelle, contrairement à une évaluation théorique telle que réalisée dans cet article.

La deuxième limite concerne les cas litigieux, dont le taux le plus important a été observé dans le modèle du CRFS (12\% de croisements). La difficulté principale résidait dans la définition du périmètre du bassin fonctionnel concerné, la disponibilité des outils et instruments d'aménagement urbain/ territorial correspondants, indispensables à la gestion des complémentarités spatio-fonctionnelles, et l'absence du cadre juridique et financier nécessaire pour ce modèle dans bien des contextes. Il est évident que pour arriver à des consensus, une démarche participative élargie devrait être engagée, intégrant des profils professionnels diversifiés et capables de garantir l'objectivité de l'analyse.

Enfin, l'essor du SAUI comme un modèle durable et résilient ne peut se concrétiser qu'à partir d'une approche opérationnelle qui dépendra fortement des capacités des acteurs locaux à intégrer tous les aspects de planification, de programmation et d'intelligence urbaines dans le cadre d'un projet alimentaire urbain (Fig. 4). Ce dernier devrait se doter d'outils (de diagnostic, de planification, d'orientation, de programmation, juridiques, etc.) qui permettent aux acteurs d'agir sur l'alimentation et sa durabilité à l'échelle de la ville ou de l'intercommunalité. Le projet devrait prendre en charge à la fois le volet biotechnique (processus du système) et d'aménagement urbain (population, équipement, infrastructures, ressources) selon une approche systémique basée sur des boucles d'équilibrage à différents niveaux (demande alimentaire et potentiel biophysique, régénération des ressources et planification aux états limites, production et agriculture urbaine, etc.). Le but étant d'optimiser le système sur le plan spatial et fonctionnel et d'assurer alors un équilibre entre demande alimentaire urbaine et potentiel biophysique. Le modèle SAUI pourrait alors être conçu comme un ensemble de boucles fonctionnelles dont l'optimisation devrait être discutée par une recherche approfondie.

\section{Conclusion}

Le travail d'évaluation de la performance des modèles alimentaires alternatifs dans la prise en charge des ODD dans l'aménagement urbain durable a donné lieu aux principaux apports suivants :

1 La proposition d'une méthode d'évaluation que nous jugeons innovante par l'association d'approches différentes (normative et opérationnelle) et plus exhaustive que les méthodes existantes à caractère plutôt thématique ou sectoriel, malgré son caractère théorique;

2 L'identification conceptuelle des profils caractéristiques des systèmes alimentaires quant à leurs objectifs et leur performance de durabilité, aussi bien dans le domaine social et environnemental qu'économique; 
$3 \mathrm{La}$ reconnaissance de l'utilité de développement du système alimentaire urbain comme nouveau modèle à part entière qui mériterait d'être formalisé et appliqué dans le domaine de programmation innovante des fonctions urbaines, de planification, de conception et d'aménagement urbain à l'aide d'outils d'ingénierie territoriale intelligente.

Ce travail débouche sur deux perspectives de recherche. La première vise à formaliser une méthode d'évaluation contextuelle de la durabilité urbaine des modèles alimentaires alternatifs par la définition d'un système de pondération ou de hiérarchisation des ODD selon les spécificités environnementales, socioéconomiques, urbaines et de gouvernance d'un contexte urbain concret. La deuxième s'intéresse à la modélisation théorique d'un SAUI et questionne sa capacité, sur le plan empirique, à améliorer la sécurité alimentaire locale. Cette dernière est explorée par nos travaux de recherche en cours, qui portent sur la ville d'Alger, et qui feront l'objet d'une prochaine publication.

\section{Matériel supplémentaire}

Annexe I. Exemples de projets SAT, CRFS et SAUI.

Annexe II. Matrice d'analyse multicritère ODD/AUD pour l'estimation de la performance urbaine du SAT en vue d'améliorer la sécurité alimentaire urbaine.

Annexe III. Matrice d'analyse multicritère ODD/AUD pour l'estimation de la performance urbaine du CRFS en vue d'améliorer la sécurité alimentaire urbaine.

Annexe IV. Matrice d'analyse multicritère ODD/AUD pour l'estimation de la performance urbaine du SAUI en vue d'améliorer la sécurité alimentaire urbaine.

Le matériel supplémentaire est disponible sur http://www. cahiersagricultures.fr/10.1051/cagri/2021019/olm.

\section{Références}

ADEME. 2020. L'évaluation environnementale dans l'industrie et les services. [11/12/2020]. https://www.ademe.fr/.

Amphoux P, Grosjean G, Salomon J. 1999. La densité urbaine, du programme au projet urbain. Rapport de recherche IREC $\mathrm{n}^{\circ} 142$. Lausanne: Institut de recherche sur l'environnement construit, département architecture, $165 \mathrm{p}$.

Angeon V, Barraud E. 2019. «Manger autrement» dans le cadre de la transition agro-écologique. Vers un système local de production et de consommation pour sécuriser l'alimentation en Guadeloupe? Socio-anthropologie (39): 115-128. https://doi.org/10.4000/socioanthropologie. 5388 .

Arib F. 2014. Les services dans l'économie verte au Maroc: opportunités de création d'emplois et défis d'innovation. Espagne, $16 \mathrm{p}$.

Aubry C, Chiffoleau Y. 2009. Le développement des circuits courts et l'agriculture périurbaine: économie et stratégies agricoles. Déméter 2013, Club Déméter, pp. 135-155.

Aubry C, Pourias J. 2013. L'agriculture urbaine fait déjà partie du métabolisme urbain. Nature et Agriculture pour la Ville. Les nouveaux désirs des citadins s'imposent, pp. 135-155.
Beischer A, Corbett J. 2016. La justice alimentaire comme réponse à la faim dans les paysages alimentaires canadiens. Comment un projet de glanage communautaire axé sur une praxis de justice alimentaire peut remédier à la dépolitisation de l'insécurité alimentaire. Justice spatiale-Spatial Justice 9: 37.

Benis K, Ferrão P. 2017. Potential mitigation of the environmental impacts of food systems through urban and peri-urban agriculture (UPA)-A life cycle assessment approach. Journal of Cleaner Production 140: 784-795. https://doi.org/10.1016/j.jcle pro.2016.05.176.

Berezowska-Azzag E. 2011. Projet urbain. Guide méthodologique. Volume 1 : Connaître le contexte de développement durable. Alger (Algérie) : Éditions Synergie, $245 \mathrm{p}$.

Berezowska-Azzag E, Abdelatif I, Akrour N, Bouallag-Azoui O, Srir M. 2014. La recherche d'intercommunalité par l'évaluation des performances environnementales locales à Alger. Méditerranée. Revue géographique des pays méditerranéens (123): 31-47. https://doi.org/10.4000/mediterranee.7366.

Blay-Palmer A, Santini G, Dubbeling M, Renting H, Taguchi M, Giordano T. 2018. Validating the City-Region Food System approach: Enacting inclusive, transformational City-Region Food Systems. Sustainability 10(5): 1680. https://doi.org/10.3390/ su10051680.

Boucher I. 2009. D'agriculture urbaine à urbanisme agricole: une participation au développement durable, une contribution à la production alimentaire. Urbanité 7.

Bousmaha A, Boulkaibet A. 2019. Planification foncière et espaces agricoles périurbains en Algérie. Le cas de l'agglomération de Skikda. Développement durable et territoires. Économie, géographie, politique, droit, sociologie 10(3). https://doi.org/10.4000/ developpementdurable.16002.

Boutefeu E. 2006. Prendre en compte le développement durable dans un projet. Guide d'utilisation de la grille RST02. Lyon (France) : Certu, $66 \mathrm{p}$.

Brand C, Bricas N, Conaré D, Daviron B, Debru J, Michel L, et al. 2017. Construire des politiques alimentaires urbaines : concepts et démarches. Versailles (France) : Éditions Quae, 160 p. https://doi. org/10.35690/978-2-7592-2618-4.

Cerema, 21 C. 2019. Pour l'appropriation de l'agenda 2030 par les collectivités française. Guide pratique, $259 \mathrm{p}$.

Charlier S, Warnotte G. 2007. La souveraineté alimentaire : regards croisés. Louvain (Belgique): Presses universitaires de Louvain, $330 \mathrm{p}$.

Charlot-Valdieu C, Outrequin P. 2004. Les méthodes et outils de la démarche $\mathrm{HQE}^{2} \mathrm{R}$. Volume $\mathrm{HQE}^{2} \mathrm{R}$. Rapport projet de recherche. Programme Énergie, Environnement et Développement durale, Commission Européenne, $36 \mathrm{p}$.

Clos J. 2018. Habitat III, la Conférence des Nations Unies axée sur les citoyens. Chronique ONU 53(3): 6-7. https://doi.org/10.18356/ c1861678-fr.

Dansero E, Pettenati G, Toldo A. 2016. Alimenter la résilience urbaine. Des nouvelles perspectives vers un plan territorial de la nourriture pour la ville de Turin. UMR 7324 CITERS. Université de Tours, pp. 31-43.

Darrot C, Bodiguel L, Noel J, Coutolleau T. 2019. La gouvernance alimentaire à l'échelle d'une agglomération: enjeux et première méthode d'approche. L'apport du projet PSDR4 FRUGAL (Formes urbaines \& gouvernance alimentaire) Interrégional Rhône-Alpes/ Grand Ouest, $4 \mathrm{p}$.

Despommier D. 2010. The vertical farm: Feeding the world in the 21st century. London (England): Macmillan, $368 \mathrm{p}$.

Deverre C, Lamine C. 2010. Les systèmes agroalimentaires alternatifs. Une revue de travaux anglophones en sciences sociales. 
Économie rurale. Agricultures, alimentations, territoires (317): 57-73. https://doi.org/0.4000/economierurale.2676.

FAO. 1996. La situation mondiale de l'alimentation et de l'agriculture. Rome (Italie).

FAO. 1999. La situation mondiale de l'alimentation et de l'agriculture. Rome (Italie).

FAO. 2006. Food Security, $4 \mathrm{p}$.

FAO. 2007. Dossier FAO pour la biodiversité. Rome (Italie).

FAO. 2012. La situation mondiale de l'alimentation et de l'agriculture. Rome (Italie).

FAO. 2014. City Region Food Systems. Sustainable food systems and urbanisation. Rome (Italie), $6 \mathrm{p}$.

FAO. 2018. Transformer l'alimentation et l'agriculture pour réaliser les ODD. 20 actions interconnectées pour orienter les décideurs. Rome (Italie), $71 \mathrm{p}$.

Forster T, Santini G, Edwards D, Flanagan K, Taguchi M. 2015. Strengthening urban rural linkages through City-Region Food Systems. Urban-Rural Linkages in Support of the New Urban Agenda 35, $19 \mathrm{p}$.

Girardin P, Bockstaller C. 1997. Les indicateurs agro-écologiques, outils pour évaluer des systèmes de culture: développement durable et recherches agronomiques. OCL. Oléagineux, corps gras, lipides 4(6): 418-426.

Guilbert S, Redlingshöfer B. 2018. Leviers de réduction des pertes et gaspillages alimentaires dans divers contextes d'évolution urbaine. Pour (4): 103-112.

Halimi R. 2014. Insertion de l'agriculture urbaine à Alger. Magister. École Polytechnique d'Architecture et d'Urbanisme d'Alger (EPAU), $137 \mathrm{p}$.

Jégou A, Augiseau V, Guyot C, Judéaux C, Monaco FX, Pech P. 2012. L'évaluation par indicateurs : un outil nécessaire d'aménagement urbain durable? Réflexions à partir de la démarche parisienne pour le géographe et l'aménageur. Cybergeo: European Journal of Geography 625. https://doi.org/10.4000/cybergeo.25600.

Kmec G. 2016. Atteindre une sécurité alimentaire durable : analyse des solutions de rechange à l'agriculture conventionnelle. Maîtrise en Environnement, Université de Sherbrooke (Canada), $96 \mathrm{p}$.

Lange KP, Korevaar G, Oskam IF, Herder PM. 2017. Developing and understanding design interventions in relation to industrial symbiosis dynamics. Sustainability 9(5): 826. https://doi.org/ $10.3390 /$ su9050826.

Lazereg M, Bellil K, Djediane M, Zaidi Z. 2020. La filière lait Algérienne face aux conséquences de la pandémie de la COVID19. Les Cahiers du Cread 36(3): 227-250.

Loconto A. 2020. Systèmes alimentaires durables. Rome: FAO, INRA, $260 \mathrm{p}$.

Loubet L. 2012. L'apprentissage de la coopération intercommunale : modalités et instruments. L'Espace Politique. Revue en ligne de géographie politique et de géopolitique (18). https://doi.org/ 10.4000/espacepolitique. 2454.

Morgan K, Sonnino R. 2010. The urban foodscape: World cities and the new food equation. Cambridge Journal of Regions, Economy and Society rsq007. https://doi.org/10.1093/cjres/rsq007.
Nations Unies. 2020. Objectifs de développement durable. [10/10/ 2020]. https://www.un.org/.

Pépin L. 2000. Les services de proximité en milieu rural québécois. Cahiers du GRIDEQ 22: 144. Rimouski.

Pradel M. 2011. Les analyses du cycle de vie dans le domaine agricole. Sciences Eaux \& Territoires 4: 4-7. https://doi.org/ $10.3917 /$ set.004.0004.

Rastoin JL. 2016. Les systèmes alimentaires territorialisés : enjeux et stratégie de développement. Journal RESOLIS 7: 12-15.

Rastoin JL, Zaite M, Ben Youssef Z. 2016. Contribution des systèmes alimentaires territorialisés à la résilience au changement climatique en région méditerranéenne-Application à un projet de cluster agro-écologique dans la vallée de Medjerda en Tunisie. Montpellier (France) : CIHEAM, 36 p.

Sala S, Ciuffo B, Nijkamp P. 2015. A systemic framework for sustainability assessment. Ecological Economics 119: 314-325. https://doi.org/10.1016/j.ecolecon.2015.09.015.

Specht K, Siebert R, Hartmann I, Freisinger UB, Sawicka M, Werner A, et al. 2014. Urban agriculture of the future: An overview of sustainability aspects of food production in and on buildings. Agriculture and Human Values 31(1): 33-51. https://doi.org/ 10.1007/s10460-013-9448-4.

Srir M. 2013. Vers un référentiel d'aménagement durable pour Alger. Urbia. Les Cahiers du Développement Urbain Durable Hors série 1: $153-171$.

Srir M. 2020. La durabilité urbaine à Alger, des enjeux locaux au référentiel d'aménagement urbain. Doctorat, EPAU, $555 \mathrm{p}$.

Tecco N, Bagliani M, Dansero E, Peano C. 2017. Toward the local territorial food system: Spaces of analysis and action. Bollettino Della Società Geografica Italiana Roma Serie XIII, X: 20-38.

Thalmann P, Doyle MR. 2013. À la recherche de la densité optimale. Green Density, pp. 135-142.

Van Der Werf H, Petit J. 2002. Évaluation de l'impact environnemental de l'agriculture au niveau de la ferme. Comparaison et analyse de 12 méthodes basées sur des indicateurs. Courrier de l'environnement de l'INRA 46: 121-133.

Verzone C, Dind JP. 2011. De l'agriculture urbaine au food urbanism : état des lieux et perspectives pour la Suisse. Urbian. Les Cahiers $d u$ Développement Urbain Durable 12: 137-160.

Viljoen A, Wiskerke JS. 2012. Sustainable food planning: Evolving theory and practice. Wageningen (The Netherlands): Wageningen Academic Publishers, 600 p. https://doi.org/10.3920/978-908686-826-1.

Wegmuller F, Duchemin E. 2010. Multifonctionnalité de l'agriculture urbaine à Montréal : étude des discours au sein du programme des jardins communautaires. [VertigO] La revue électronique en sciences de l'environnement 10(2). https://doi.org/10.4000/ver tigo.10445.

Zahm F, Ugaglia AA, Barbier JM, Boureau H, Del'Homme B, Gafsi M, et al. 2019. Évaluer la durabilité des exploitations agricoles : la méthode IDEA v4, un cadre conceptuel combinant dimensions et propriétés de la durabilité. Cahiers Agricultures 28: 5. https://doi. org/10.1051/cagri/2019004.

Citation de l'article : Saci H, Berezowska-Azzag E. 2021. Sécurité alimentaire et durabilité urbaine des modèles alimentaires alternatifs : analyse multicritère basée sur les objectifs de développement durable et l'aménagement urbain durable. Cah. Agric. 30 : 35. 